\title{
An Effectual Approach to Reduce the Noise in ECG
}

\author{
Giritharan Ravichandran ${ }^{[1]}$, \\ E.G.S.Pillay Engineering College.
}

\begin{abstract}
Electrocardiogram (ECG) represents electrical activity of human heart. ECG is composite from 5 waves - $P, Q, R, S$ and $T$. This signal could be measured by electrodes from human body in typical engagement. Signals from these electrodes are brought to simple electrical circuits with amplifiers and analogue - digital converters. Abnormal patterns of ECG may be due to interference from power line which is easily recognizable since interfering voltage in the ECG would have frequency of $60 \mathrm{~Hz}$. This interference due to stray effect of alternating current on the patient or because of alternating current fields produce in the patient cable, and white noise generated by ECG equipment and these artifacts which must be removed otherwise it will convey an incorrect information regarding the patient's heart condition. The work in this paper is the design of FIR digital filters with new Window to remove the interferences or the artifacts and calculated SNR of noiseless ECG and compared with existing windows. Three filters are considered: Low pass, high pass and band reject filters. Each filter is used to filter the raw noisy ECG signal and results are observed and tabulated. In this paper we apply a new windowing technique to improve the SNR of ECG, so as to view the ECG noise-free.
\end{abstract}

Keywords: Window, Window Functions, Window Coefficients, SNR

\section{Introduction}

Electrocardiography (ECG) is the acquisition of electrical activity of the heart captured over time by an external electrode attached to the skin. Each of the cell membranes that form the outer covering of the heart cell has an associated charge which is depolarized during every heartbeat. These appear as tiny electrical signals on the skin which can be detected and amplified by the ECG.

Signal processing is very important and evident tool in fields of biomedical engineering. The biomedical signal processing field has advanced to the stage of practical application of signal processing and pattern analysis techniques for efficient and improved non-invasive diagnosis, online monitoring of critical patients, and rehabilitation. ECG signal is a graphical representation of cardiac activity and it used to measure the various cardiac diseases and abnormalities present in heart. ECG signals are Composed of P wave, QRS complex, $\mathrm{T}$ wave and any deviation in these parameters indicate abnormalities present in heart. The standard ECG signal is shown in Fig. 1.

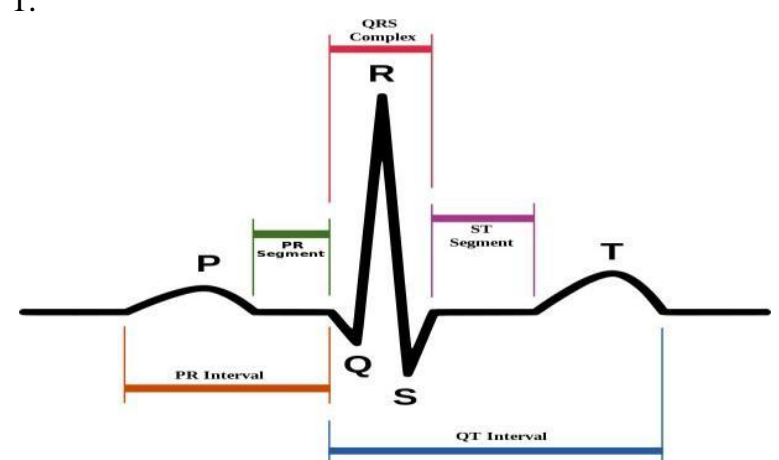

Fig1:- An standard ECG waveform (source: Google images)

Signal processing is a huge challenge since the actual signal value will be $0.5 \mathrm{mV}$ in an offset environment of $300 \mathrm{mV}$. Other factors like AC power-supply interference, RF interference from surgery equipment, and implanted devices like pace makers and physiological monitoring systems can also impact accuracy. The main sources of noise in ECG are

- Baseline wander (low frequency noise)

- Power line interference ( $50 \mathrm{~Hz}$ or $60 \mathrm{~Hz}$ noise from power lines)

- Muscle noise (This noise is very difficult to remove as it is in the same region as the actual signal. It is usually corrected in software.)

- Other interference (i.e., radio frequency noise from other equipment). 


\section{Previous Techniques}

Digital FIR filters are successfully employed in processing electrocardiographic [1] signals for measurement. ECG which is a biomedical signal is naturally corrupt by various interferences such as $50 / 60 \mathrm{~Hz}$ Power Line Interferences (PLI) and some other biomedical signals like baseline wander ECG signal frequency is approximately between $0.05 \mathrm{~Hz}$ and $100 \mathrm{~Hz}$. Baseline Wander frequency is below $1 \mathrm{~Hz}$. These interferences have to be removed from ECG signal in order to obtain correct clinical information of the heart. Since the frequency of ECG depends on the muscle movement rate and pressure it can be reduced to the barest minimum during ECG measurement by the patient staying still and quiet so that the muscles are fully relaxed. The normal ECG signal is shown in Fig 2.

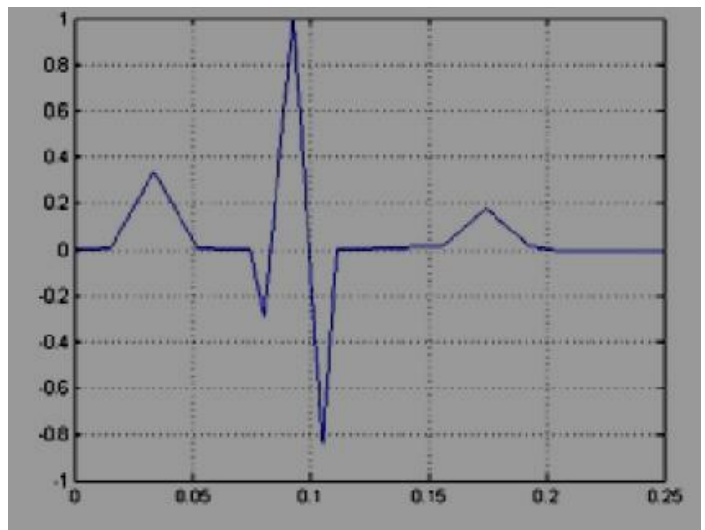

Fig.2 : Normal ECG waveform

\section{Window Functions}

In this section we present different window methods which are used to design of FIR filters.[2]

Hamming window:

$$
\begin{aligned}
w_{h} & =0.54-0.46 \cos \left(\frac{2 \pi n}{N-1}\right) \quad \text { for } 0<n<N-1 \\
& =0 \text { other wise }
\end{aligned}
$$

\section{Hanning window}

$$
\begin{aligned}
w_{h}= & 0.5-0.5 \cos \left(\frac{2 \pi n}{N-1}\right) \quad \text { for } 0<n<N-1 \\
= & 0 \text { other wise }
\end{aligned}
$$

\section{Kaiser window}

Kaiser window has very desirable characteristics bothin time domain and frequency domain. A good window should be a time limited function with a Fourier transform that is band limited and Kaiser Window possesses such characteristics closely. Kaiser window is defined by the expression,

$\omega_{k}(\beta, n)=\frac{\beta\left[1-. .\left(\frac{2 n}{N-1}\right)^{2}\right]^{1 / 2}}{J_{0} \beta}$

$$
\begin{aligned}
& \frac{(\mathrm{N}-1)}{2}<=\mathrm{n}<=\frac{(\mathrm{N}-1)}{2} \\
& \text { or }=0 \text { otherwise }
\end{aligned}
$$

Where $\mathrm{N}$ is the order of the filter and $\mathrm{Jo}(\mathrm{x})$ is the modified Bessel function of the first kind of order zero, and is given by,

$$
j_{0}(p)=1+\sum_{k-0}^{-}\left[\left(\frac{p}{2}\right)^{k} / k !\right]^{2}
$$

$$
\text { (2) }
$$

From (2), for $\mathrm{k}=0$ $\mathrm{J} 0(\mathrm{P})=1+\ldots$.

Alternatively, $\mathrm{J} 0(\mathrm{P})$ can be written as 
$j_{0}(p)=1+\sum_{k-1}^{-}\left[\left(\frac{p}{2}\right)^{k} / k !\right]^{2}$

In most cases the upper limit of $\mathrm{k}$ turns out to be $\mathrm{k}=9$ or 10 Experience shows that as the Parameter $\mathrm{B}$ is varied in both the transition bandwidth of a filter and the peak ripple in the side lobes change. The filter designer can therefore trade off main-lobe width for side-lobe ripple amplitude. Typical values of $\beta$ lies in the range of: $\quad 4<\beta<11$ The value of $B$ to be used in any design depends on such factors like the order of the filter, the type of signal to be filtered and targeted signal to noise ratio. A typical Kaiser Window function is depicted as fig below.

\section{Proposed Window:}

Proposed window is designed based on Clonal selection algorithm is based on the clonal selection principle in the artificial immune system.

a) Randomly generate a repertoire of candidate antibodies $A b=A b\{r\} U A b\{m\}(r+m=N)$, where $A b\{r\}$ is the remaining repertoire, while $\mathrm{Ab}\{\mathrm{m}\}$ is the memory repertoire;

b) Determine the affinity $\mathrm{f}$ of each antibody in $\mathrm{Ab}$ in relation to the antigen $\mathrm{Ag}$;

c) Select $n$ highest affinity antibodies in $A b$ to compose a new repertoire $\operatorname{Ab}\{n\}$;

d) The $\mathrm{n}$ selected antibodies will be cloned independently and proportionally to their antigenic affinity, generating a temporary repertoire $\mathrm{C}$ of clones;

e) The repertoire $\mathrm{C}$ is submitted to an affinity maturation process inversely proportional to the antigenic affinity, generating a population $\mathrm{C}^{*}$ of matured clones;

f) Determine the affinity $\mathrm{f} *$ between each antibody in $\mathrm{C}^{*}$ and the antigen $\mathrm{Ag}$;

g) From the matured clones $C^{*}$, reselect improved antibody $\mathrm{Ab}^{*}$ to update the memory repertoire $\mathrm{Ab}\{\mathrm{m}\}$;

h) Replace the d lowest affinity antibodies in $\mathrm{Ab}\{\mathrm{r}\}$ with random antibodies $\mathrm{Ab}\{\mathrm{d}\}$;

i) Check whether there is an antibody which can meet certain requirements. If there is one, quit this process; else go to step b) to continue the search iterations. The programs run on PC with $3.2 \mathrm{G} \mathrm{CPU}$. The experiments repeated for ten times and came down to similar results. The average running time is $14.12 \mathrm{~s}$, and the mean iteration times are 230. Choose one of the simulated results as $\mathrm{a} \square[0.5154,0.4711,0.0135]$, the corresponding window function is

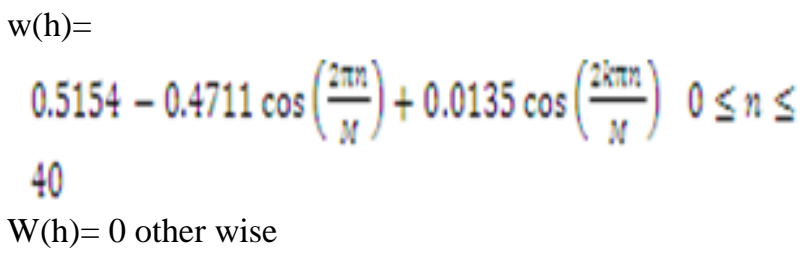

\section{Design of Filters using Windows}

The low pass filter removes the high frequencies inECG signal. The order of the filter is 25 .

Steps to design Low pass filter:

1) Lower cut-off frequency: $80 \mathrm{~Hz}$

2) Sampling frequency $: 500 \mathrm{~Hz}$

3) The desired transfer function of high pass filter is

$h_{d}(n)=\frac{\sin 0.32 \pi n}{\pi n}$

4) Let $w(n)=$ Transfer function of window

5) The transfer function of window based low pass filter is $h(n)=h_{d}(n) * w(n)$

The Time response and frequency response is shown in below Fig3.

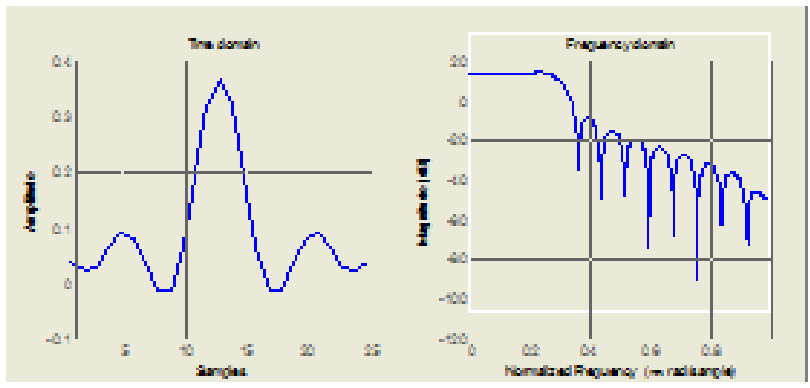

Fig 3: Response of the low pass filter 
Steps to design high pass filter:

1) Lower cut-off frequency: $0.5 \mathrm{~Hz}$

2) Sampling frequency : $500 \mathrm{~Hz}$

3) The desired transfer function of high pass filter is

4) Let $w(n)=$ Transfer function of window

5) The transfer function of window based high pass filter is $h(n)=h_{d}(n) * w(n)$

The Time response and frequency response is shown in below Fig 4.

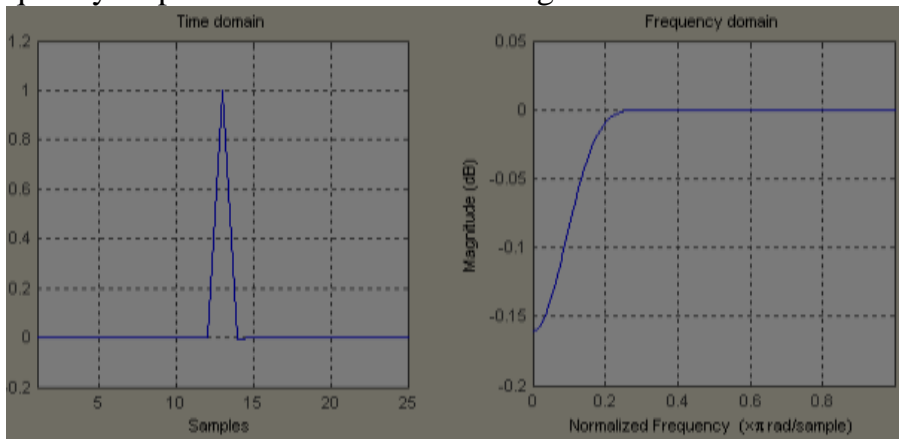

Fig 4.: Response of the high pass filter

\section{Design of Band Reject Filter}

The Band Reject filter removes the corrupting power line frequency noise in ECG signal. The power line frequency is $560 \mathrm{~Hz}$ and sampling frequency is $500 \mathrm{~Hz}$. The order of the filter is 25 .

\section{Steps to design Band reject filter:}

1) Lower cut-off frequency: $59.5 \mathrm{~Hz}$

2) Upper cut-off frequency: $60.5 \mathrm{~Hz}$

3) Sampling frequency :500 Hz

4) The desired transfer function of high pass

filter is

$$
h_{d}(n)=\frac{\sin \pi n+\sin 0.238 \pi n-\sin 0.242 \pi n}{\pi n}
$$

5) Let $w(n)=$ transfer function of Kaiser window.

6) The transfer function of window based high pass filter is $h(n)=h_{d}(n) * w(n)$

The magnitude and frequency response of Band reject filter is shown below.

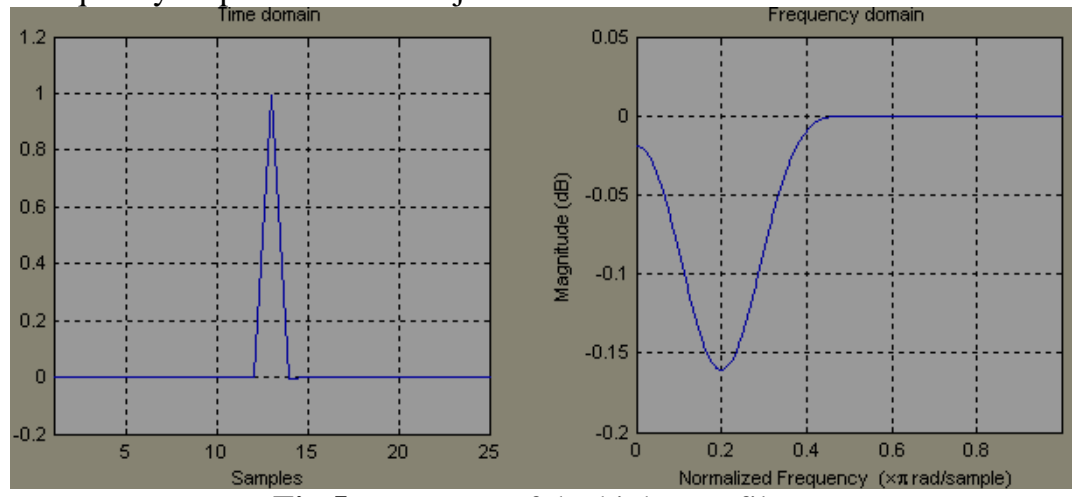

Fig 5: Response of the high pass filter

\section{Design of Band Reject Filter}

The Band Reject filter removes the corrupting power line frequency noise in ECG signal. The power line frequency is $560 \mathrm{~Hz}$ and sampling frequency is $500 \mathrm{~Hz}$. The order of the filter is 25 .

\section{Steps to design Band reject filter:}

1) Lower cut-off frequency: $59.5 \mathrm{~Hz}$ 
2) Upper cut-off frequency:60.5 Hz

3) Sampling frequency :500 Hz

4)The desired transfer function of high pass

filter is

$h_{d}(n)=\frac{\sin \pi n+\sin 0.238 \pi n-\sin 0.242 \pi n}{\pi n}$

5)Let $w(n)=$ transfer function of Kaiserwindow.

6)The transfer function of window basedhigh pass filter is $h(n)=h_{d}(n) * w(n)$

The magnitude and frequency response of Band reject filter is shown below.

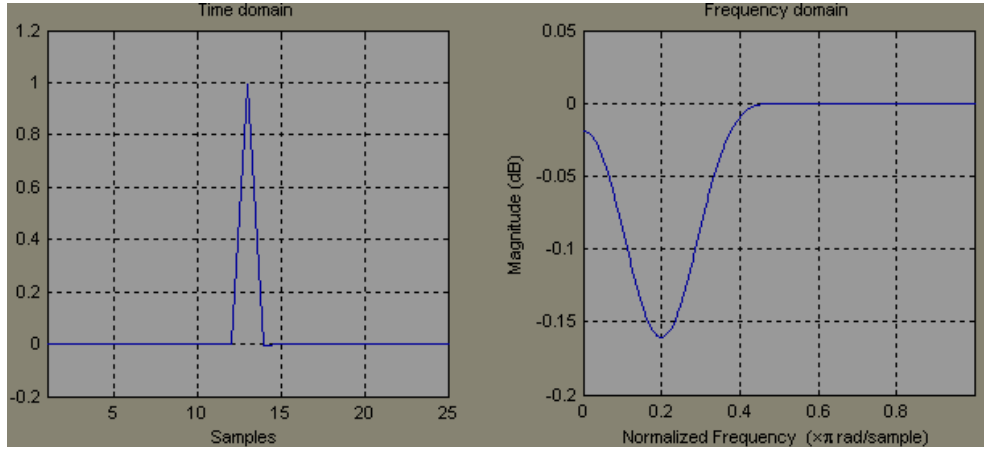

Fig. 7: Frequency response of the Band reject filter

\section{Results:}

The results shows responses of the cascaded lowpass, high pass filter and Band reject filter and compares the signal to noise ratio of ECG signal before and after the filtering for different window methods.

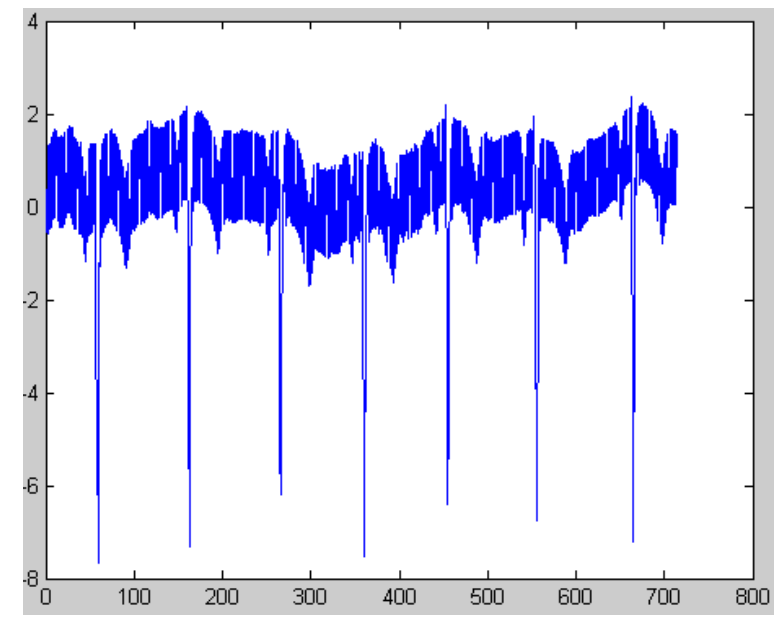

Fig. 7:Noised ECG signal

When the raw ECG signal of Fig 7 is filtered with three filters in cascade i.e. hamming, hanning and Kaiser windows the responses are obtained as shown in Figures 8,9,10 respectively. Fig 11 shows response of filters with proposed window given by which shows improved SNR of ECG compared to existing windows.. The cascaded arrangement is shows how digital filters are connected in an electrocardiograph which is an instrument for checking the heart conditions of patients. 


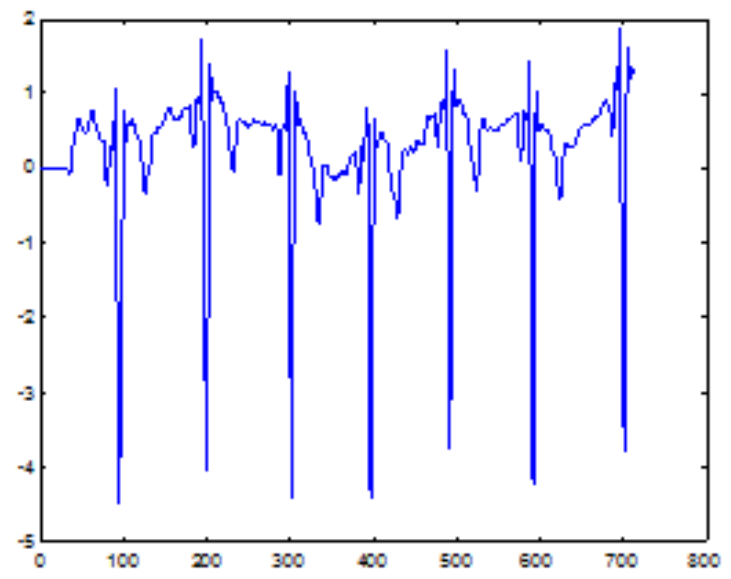

Fig 8: Response of ECG for hamming window

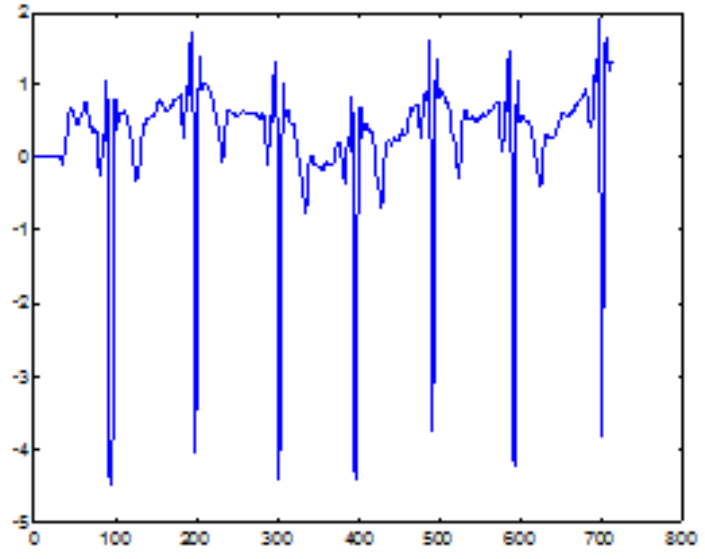

Fig 9: Response of ECG for hanning window

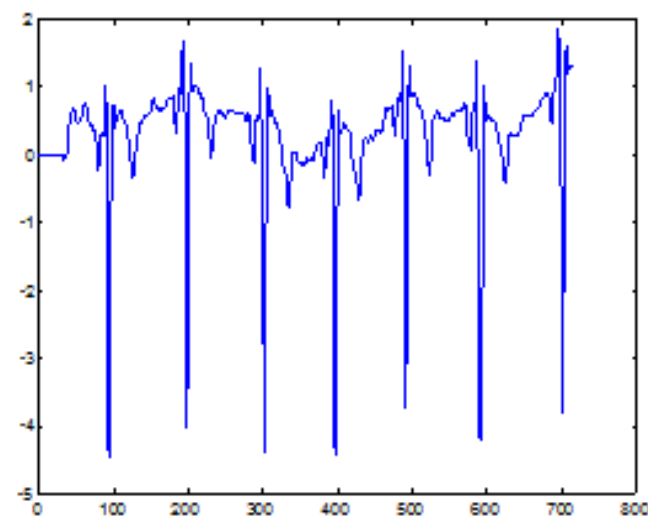

Fig 10: Response of ECG for Kaiser Window 


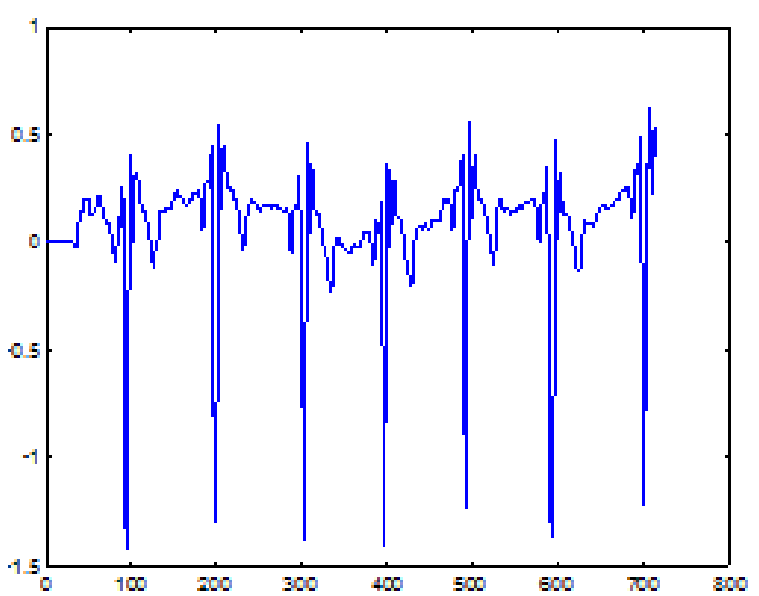

Fig 11: Response of ECG for proposed window

\begin{tabular}{|l|c|}
\hline \multicolumn{1}{|c|}{ Window } & SNR of filtered ECG \\
\hline Hamming & 0.9431 \\
\hline Hanning & 0.9454 \\
\hline Kaiser & 0.9421 \\
\hline Proposed Window & $\mathbf{2 8 . 5 6 7}$ \\
\hline
\end{tabular}

Table 1: Summary of the implementation results of the three digital filters designed with different Windows.

\section{Conclusion:}

In this paper we are using different windows for design of FIR filters to remove power line interferences $60 \mathrm{~Hz}$ of ECG signal. Here we have attained different SNR values for existing windows and proposed window which shows clearly better SNR values as compared to remaining windows as in Table1.

Also other methods of improving strength of ECG resulted to maximum SNR of 25. Comparing to this, the technique used here gives the better results.

\section{Acknowledgements:}

I wish to thank my father Dr. MG. Ravichandran and my mother Dr. G. Ayyappakumari for their keen support and encouragement. I also wish to thank my Head Of the Department Mrs. R. Lakshmi Rekha for giving me a sense of light to this topic, and her strong support.

\section{Reference:}

[1]. Hand book of biomedical instrumentation - second edition- Khandpur-Tata McGraw-Hill

[2]. Digital signal processing - Principles, Algorithms, and Applications- John G.Proakis

[3]. Digital signal processing -Ramesh babu.

[4]. Mitov I. P; -A method for reduction of power line interference in the ECGll. Medical Engineering Physics, vol. 26, no. 10, pages 879-887,December,2004

[5]. Sarkar, N. (2003): Elements of digital signal Processing: Khanna Publishers, Delhi India.

[6]. A Novel FIR Filter Design Based on Clonal Selection Algorithm Qiusheng Wang*, ShaokunGao, Jingpo Zhao School of Automation Science and Electrical Engineering [7] Xu Yuan, Yang bo, Zhu Mingcheng, " A New Genetic Algorithm Involving Mechanism of Simulated Annealing for Digital FIR Evolving Hardware Algorithm," Journal of Computer-aided Design \& Computer Graphics. 18(5), 674-679 (2006).

[7]. CHEN Min-ge -Design of Equiripple Linear-Phase FIR Filters Using MATLAB\| Control, Automation and Systems Engineering (CASE), 2011 International Conference.

[8]. Mahdi Mottaghi-Kashtiban and Mahrokh G. Shayesteh, - A New Window Function for Signal Spectrum Analysis and FIR Filter Design\| Electrical Engineering (ICEE), 2010 18th Iranian Conference.

[9]. A. Oppenheim, R. Schafer, and J. Buck, Discrete-Time Signal Processing, fifth printing, Prentice- Hall, 1998 pp 444.

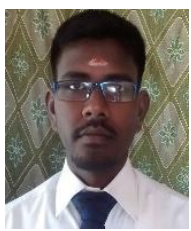

Giritharan Ravichandran is a $3^{\text {rd }}$ year B.E (ECE) student in E.G.S.Pillay Engineering College, Nagapattinam. He has published 2 papers in International Journal (IJARECE), and presented papers in national and international conferences. His interest areas include BrainBrain Interface, Image Processing, Digital signal Processing, Medical and Wearable Electronics. 